\title{
Graded ferroelectric capacitors with robust temperature characteristics
}

\author{
Mohamed Y. El-Naggar, ${ }^{\text {a) }}$ Kaushik Dayal, David G. Goodwin, and Kaushik Bhattacharya \\ Division of Engineering and Applied Science, California Institute of Technology, Pasadena, \\ California 91125
}

(Received 15 June 2006; accepted 19 August 2006; published online 13 December 2006)

\begin{abstract}
Ferroelectric thin films offer the possibility of engineering the dielectric response for tunable components in frequency-agile rf and microwave devices. However, this approach often leads to an undesired temperature sensitivity. Compositionally graded ferroelectric films have been explored as a means of redressing this sensitivity, but experimental observations vary depending on geometry and other details. In this paper, we present a continuum model to calculate the capacitive response of graded ferroelectric films with realistic electrode geometries by accurately accounting for the polarization distribution and long-range electrostatic interactions. We show that graded $c$-axis poled $\mathrm{Ba}_{x} \mathrm{Sr}_{1-x} \mathrm{TiO}_{3}$ (BST) parallel plate capacitors are ineffective while graded $a$-axis poled BST coplanar capacitors with interdigitated electrodes are extremely effective in obtaining high and temperature-stable dielectric properties. (0) 2006 American Institute of Physics.
\end{abstract}

[DOI: $10.1063 / 1.2369650]$

\section{INTRODUCTION}

Ferroelectric thin films, especially those made of $\mathrm{Ba}_{x} \mathrm{Sr}_{1-x} \mathrm{TiO}_{3}$ (BST), have recently emerged as candidate materials for tunable components in frequency-agile RF and microwave devices that benefit from their high dielectric constants and related high tunability. ${ }^{1-6}$ However, the dielectric behavior of these materials depends very strongly on temperature near the Curie temperature $T_{C}$. This means that, for conventional ferroelectrics, the dielectric properties of the tunable devices may drift strongly depending on ambient conditions. In tunable filters, for instance, this would be unacceptable since the resonant frequency varies directly with changes in the dielectric constant. ${ }^{7}$

Currently, only few materials satisfy the stringent requirements of low losses and temperature stability in cellular base stations and handheld devices. These materials are in the low to medium dielectric constant range. ${ }^{8}$ In short, ferroelectrics are both highly tunable and possess high dielectric constants, but an equally important challenge emerges: the need for a low temperature dependence of the dielectric constant over the operating temperature range. ${ }^{9}$

Compositionally graded $\mathrm{Ba}_{x} \mathrm{Sr}_{1-x} \mathrm{TiO}_{3}$ thin films have been investigated as a means of overcoming this temperature instability. The Curie temperature spans a broad range, depending on composition. Therefore, the idea is that the heterogeneous structure would result in a diffuse phase transition and a high dielectric constant over a wide range of temperatures corresponding to different Curie temperatures for the different regions. Recent experimental efforts have established the promise of this approach ${ }^{7,10-12}$ and modeling efforts have followed in this direction. ${ }^{13,14}$ The models solve for the polarization of one-dimensional systems and subsequently (separately) compute the effective dielectric behavior by summing capacitances. However, experimental results also show the importance of capacitor geometry. ${ }^{12}$ The place-

${ }^{a)}$ Electronic mail: mnaggar@caltech.edu ment of the electrodes and the compositional grading give rise to a complex coupling between the polarization and the electrostatic fields. Therefore, there is a need to extend this to more complex systems by taking into account the electrostatic fields, polarization distribution, and geometry in an integrated manner.

This motivates us to address the graded thin film problem using a continuum model that takes into account the spatial variation in properties and the long-range electrostatic interactions. We present an integrated model that allows us to compute the built-in electric potential, polarization gradient, and electrode charges both in the presence and absence of externally applied electrical field. This approach enables the direct calculation of the temperature-dependent dielectric behavior for different compositional gradings and device geometries. We seek an understanding of the combinatorial behavior of ferroelectric heterostructures and provide results that are useful as design tools for the emerging functionally graded devices.

\section{MODEL}

We start by considering the continuum energy of a ferroelectric crystal ${ }^{15}$ occupying a region $\Omega$ in space,

$$
E=\int_{\Omega}[U(\nabla \mathbf{p})+W(\mathbf{p}, \mathbf{x}, T)] d \Omega+\frac{\epsilon_{o}}{2} \int_{\mathbb{R}^{3}}|\nabla \phi|^{2} d V .
$$

Here, $U$ is the energy density associated with the presence of a polarization gradient and we take it to be of the form $U(\nabla \mathbf{p})=\left(a_{0} / 2\right)|\boldsymbol{\nabla} \mathbf{p}|^{2}$. The constant $a_{0}$ is related to the physical dimension across which polarization changes take place and is set to $10^{-9} \mathrm{~V} \mathrm{~m}^{3} \mathrm{C}^{-1}$, corresponding to a few nanometers. ${ }^{16} \mathrm{~W}$ is the Devonshire-Ginzburg-Landau (DGL) energy density expanded in powers of the polarization $\mathbf{p}$, the coefficients being functions of temperature and composition, i.e., position in a graded film, 
(a)
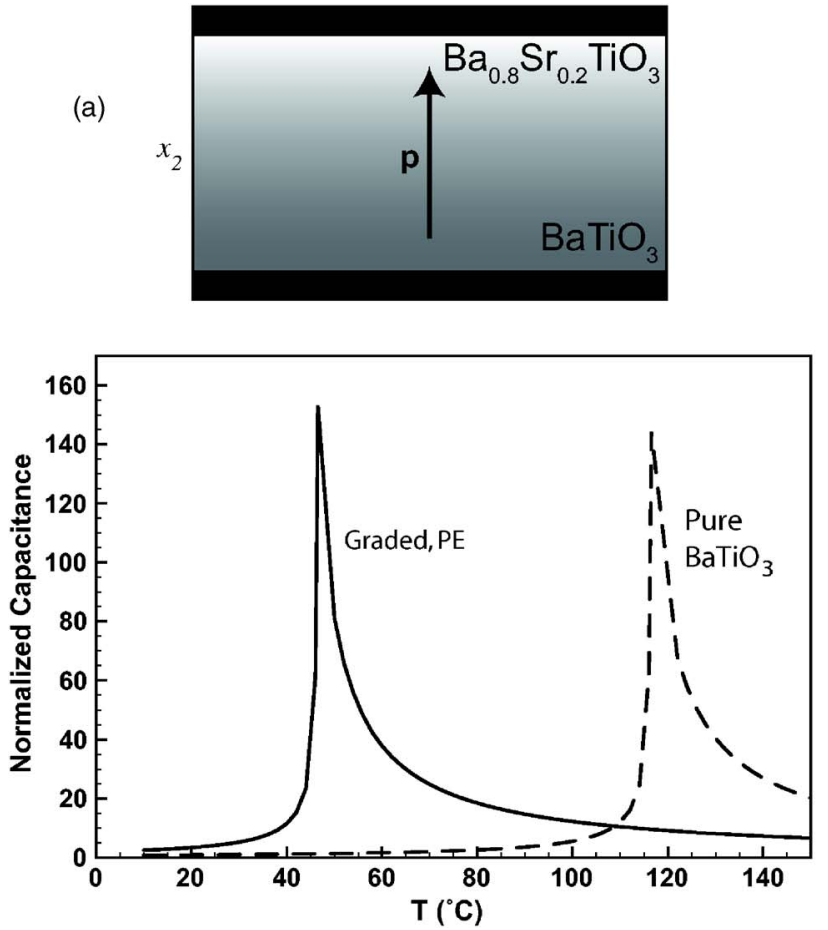

(b)
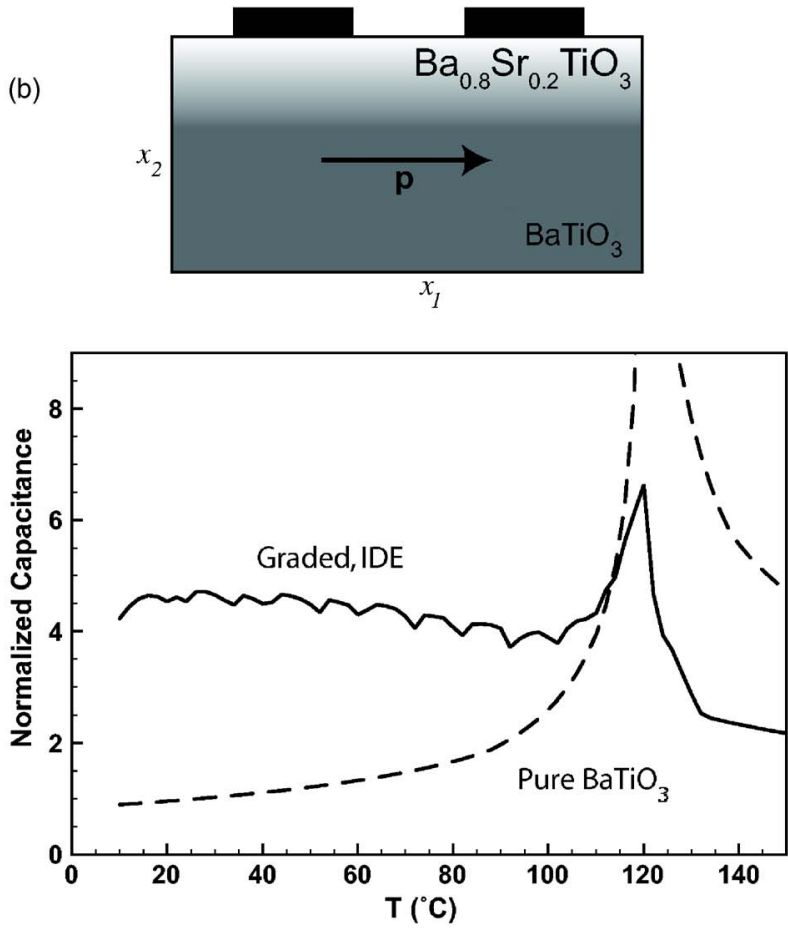

FIG. 1. (Color online) The dielectric response vs temperature for two cases of $200 \mathrm{~nm}$ graded $\mathrm{BaTiO}_{3}-\mathrm{Ba}_{0.8} \mathrm{Sr}_{0.2} \mathrm{TiO}_{3}$ capacitors: (a) parallel electrodes ( $\mathrm{PEs}$ ) and (b) interdigitated electrodes (IDEs). The desired stability is achieved for a wide range of operating temperatures in the IDE geometry with an $a$-axis film. Moreover, the capacitance is enhanced by a factor of $\sim 5$ compared to the already high capacitance of pure $\mathrm{BaTiO}_{3}$.

$$
\begin{aligned}
W= & \frac{a_{1}\left(x_{2}, T\right)}{2}\left(p_{1}^{2}+p_{2}^{2}\right)+\frac{a_{2}\left(x_{2}, T\right)}{2}\left(p_{1}^{4}+p_{2}^{4}\right)+\frac{a_{3}\left(x_{2}, T\right)}{2} p_{1}^{2} p_{2}^{2} \\
& +\frac{a_{4}\left(x_{2}, T\right)}{2}\left(p_{1}^{6}+p_{2}^{6}\right)+\frac{a_{5}\left(x_{2}, T\right)}{4} p_{1}^{4} p_{2}^{4},
\end{aligned}
$$

where $x_{2}$ is along the thickness direction of the film and $p_{1}$ and $p_{2}$ are the two components of polarization. The last term in Eq. (1) is the electrostatic energy associated with the electric field $\mathbf{E}=-\boldsymbol{\nabla} \phi$ and can be obtained by solving Gauss's equation,

$$
\boldsymbol{\nabla} \cdot\left(\mathbf{p}-\epsilon_{o} \boldsymbol{\nabla} \phi\right)=0 \text { in } \mathbb{R}^{3},
$$

with voltage specified at the electrodes and decaying at infinity in the surrounding medium. By taking the first variation of the energy to be zero: ${ }^{17}$

$\boldsymbol{\nabla} \cdot\left(\frac{\partial U}{\partial(\boldsymbol{\nabla} \mathbf{p})}\right)-\frac{\partial W}{\partial \mathbf{p}}-\boldsymbol{\nabla} \phi=0$ in $\Omega$ with $\boldsymbol{\nabla} \mathbf{p} \cdot \mathbf{n}=0$ on $\partial \Omega$.

Equations (3) and (4) represent the equilibrium equations of our system and we need to solve them simultaneously for a given geometry and composition distribution.

Of special interest is the effect of capacitor geometry, since experiments suggest different dielectric behaviors with temperature for the parallel plate and interdigitated electrode configurations. ${ }^{12}$ Here we consider two geometries illustrated schematically in Fig. 1. The first is a film graded along the entire thickness of the film and completely shielded by two parallel plate electrodes (PEs). The second is a graded film with interdigitated electrodes (IDEs), and the compositional grading occurs in the region where the electric field pen- etrates into the film. In both cases, PE and IDE, we look for solutions with the polarization aligned with the applied nominal applied field and use the same range of compositions.

For the PE configuration in one dimension, $W$ from Eq. (2) becomes

$$
W=\frac{a_{1}\left(x_{2}, T\right)}{2} p_{2}^{2}\left(x_{2}\right)+\frac{a_{2}\left(x_{2}, T\right)}{4} p_{2}^{4}\left(x_{2}\right)+\frac{a_{4}\left(x_{2}, T\right)}{6} p_{2}^{6}\left(x_{2}\right),
$$

and Eqs. (3) and (4) are applied in one dimension to become

$$
\begin{gathered}
a_{o} \frac{d^{2} p_{2}}{d x_{2}^{2}}-a_{1}\left(x_{2}, T\right) p_{2}\left(x_{2}\right)-a_{2}\left(x_{2}, T\right) p_{2}^{3}\left(x_{2}\right)-a_{4}\left(x_{2}, T\right) p_{2}^{5}\left(x_{2}\right) \\
-\frac{d \phi}{d x_{2}}=0
\end{gathered}
$$

with $\frac{d p_{2}}{d x_{2}}(0)=0$ and $\frac{d p_{2}}{d x_{2}}(t)=0$

$\frac{d p_{2}}{d x_{2}}-\epsilon_{o} \frac{d^{2} \phi}{d x_{2}^{2}}=0 \quad$ with $\phi(0)=0$ and $\phi(t)=\phi_{t}$,

where $t$ is the thickness of the film. The bottom electrode is kept grounded while a potential $\phi_{t}$ is imposed on the top plate.

For the IDE configuration, the ferroelectric is not completely shielded, allowing electric fields to be generated outside the device. Under these conditions, the electrostatics must be solved over all of space. Equations (3) and (4) are applied in two dimensions with a boundary element tech- 
TABLE I. DGL coefficients for $\mathrm{BaTiO}_{3}, \mathrm{SrTiO}_{3}$, and $\mathrm{PbTiO}_{3}$ used in this report (Refs. 13 and 17-19). All coefficients given in SI units and temperature in ${ }^{\circ} \mathrm{C}$.

\begin{tabular}{cccc}
\hline \hline Coefficient & $\mathrm{BaTiO}_{3}$ & $\mathrm{SrTiO}_{3}$ & $\mathrm{PbTiO}_{3}$ \\
\hline$a_{1}$ & $6.6 \times 10^{5}(T-110)$ & $1.41 \times 10^{6}(T+253)$ & $9.04 \times 10^{5}(T-479)$ \\
$a_{2}$ & $1.44 \times 10^{7}(T-175)$ & $8.4 \times 10^{9}$ & $-3.116 \times 10^{8}$ \\
$a_{3}$ & $3.94 \times 10^{9}$ & $3.94 \times 10^{9}$ & $3.94 \times 10^{9}$ \\
$a_{4}$ & $3.96 \times 10^{10}$ & $3.96 \times 10^{10}$ & $1.584 \times 10^{9}$ \\
$a_{5}$ & $2.39 \times 10^{14}$ & $2.39 \times 10^{14}$ & $2.39 \times 10^{14}$ \\
\hline \hline
\end{tabular}

nique that enables us to address realistic device geometries. $^{20,21}$

The heterogeneity of the ferroelectric is taken into account by making $a_{1}, a_{2}$, and $a_{4}$ spatially dependent in the equations above. Table I lists the coefficients for $\mathrm{BaTiO}_{3}$, $\mathrm{SrTiO}_{3}$, and $\mathrm{PbTiO}_{3}$. We linearly interpolate for the intermediate compositions $(0 \leqslant x \leqslant 1)$ in the graded structure while $a_{3}$ and $a_{5}$ are chosen to retain a reasonable energy barrier for polarization switching. ${ }^{17}$

The procedure outlined here allows us to calculate the polarization and electric field at any point. To calculate the dielectric behavior, one can compute the charge induced on the ferroelectric/metal boundary. For the PE geometry

$$
\sigma_{0}=\left(p_{2}-\epsilon_{o} \frac{d \phi}{d x_{2}}\right)_{x_{2}=0}, \quad \sigma_{t}=\left(\epsilon_{o} \frac{d \phi}{d x_{2}}-p_{2}\right)_{x_{2}=t} .
$$

The difference in free charge, $\Delta \sigma$, between zero and small applied field $\phi_{t}$ is used to compute the capacitance at a specific temperature. The capacitance is similarly calculated for the IDE geometry at the specific locations of the coplanar electrodes.

\section{RESULTS AND DISCUSSION}

We now examine the results for a $200 \mathrm{~nm}$ pure $\mathrm{BaTiO}_{3}$ film (as a test of the procedure and for reference) as well as a $200 \mathrm{~nm}$ graded BST thin film. The endpoints of compositional grading are $\mathrm{BaTiO}_{3}$ at the bottom and $\mathrm{Ba}_{0.8} \mathrm{Sr}_{0.2} \mathrm{TiO}_{3}$ at the top. In the PE geometry, the grading extends linearly from one electrode to another, while in the IDE configura- tion, this linear grading occupies the top third of the film, which is the extent of significant field penetration under the chosen electrode/grading geometry. The temperature dependence of the capacitance is presented in Fig. 1, for both configurations of graded films. The calculated capacitance is normalized by the room temperature capacitance of $\mathrm{BaTiO}_{3}$ due to the ambiguity of defining a common intensive quantity such as dielectric constant to compare both geometries. Therefore, we choose to normalize the results such that the normalized capacitance of pure $\mathrm{BaTiO}_{3}$ is unity at room temperature, for both cases.

In both configurations, the method produces the expected transition temperature and dielectric peak for pure $\mathrm{BaTiO}_{3}$ at $120{ }^{\circ} \mathrm{C}$. The graded film behavior, however, differs for the PE and IDE geometries. The PE graded film merely experiences a temperature shift of the dielectric peak, such that the transition occurs between the Curie temperatures of the two extreme compositions. In other words, the parallel plate geometry in combination with a $c$-axis film does not lead to a broadening of the phase transition, but the film behaves like a conventional ferroelectric, with a $T_{C}$ intermediate between those of the composition endpoints. That is not the case for the IDE graded film, where the capacitance displays a high and flat response over a wide temperature range. The compositional grading with the IDE geometry results in the desired broad transition. Small irregularities can be seen in the dielectric response due to the approximation of a linear grading as a series of step changes in composition. This physically corresponds to a multilayer ferroelectric film. These small suppressed transitions are physical and have been found experimentally. ${ }^{10}$

This result emphasizes the influence of the geometry on the dielectric behavior, specifically for a system where the electrostatics plays a major role. To examine this effect in detail, we now study the computed polarization and electric potential for both geometries, under zero applied field and room temperature. The field in the film is an inherently built-in one and is commonly referred to in the literature as the depolarizing field. The computed polarization and potential profiles are displayed in Fig. 2 for the BST graded films of both geometries. In the PE geometry [Fig. 2(a)], the po-

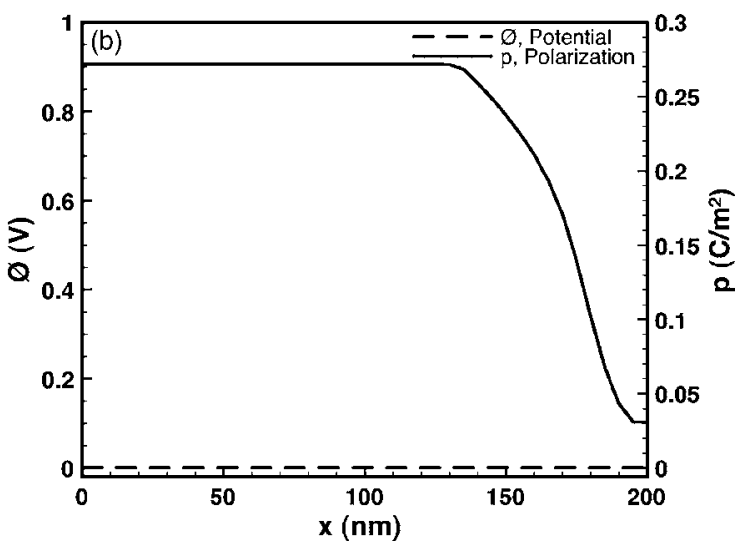

FIG. 2. Calculated room temperature spontaneous polarization and built-in potential for (a) $200 \mathrm{~nm}$ graded $\mathrm{BaTiO}_{3}-\mathrm{Ba}_{0.8} \mathrm{Sr}_{0.2} \mathrm{TiO}_{3}$ thin film used in the PE geometry and (b) $200 \mathrm{~nm}$ graded $\mathrm{BaTiO}_{3}-\mathrm{Ba}_{0.8} \mathrm{Sr}_{0.2} \mathrm{TiO}_{3}$ film used in the IDE geometry. In the PE geometry, the compositional grading does not lead to a polarization gradient due to the strong electrostatic interactions manifested by the large built-in potential. A polarization gradient is achieved in the IDE capacitor corresponding to multilayer parallel capacitances with weak interactions between the different layers. 


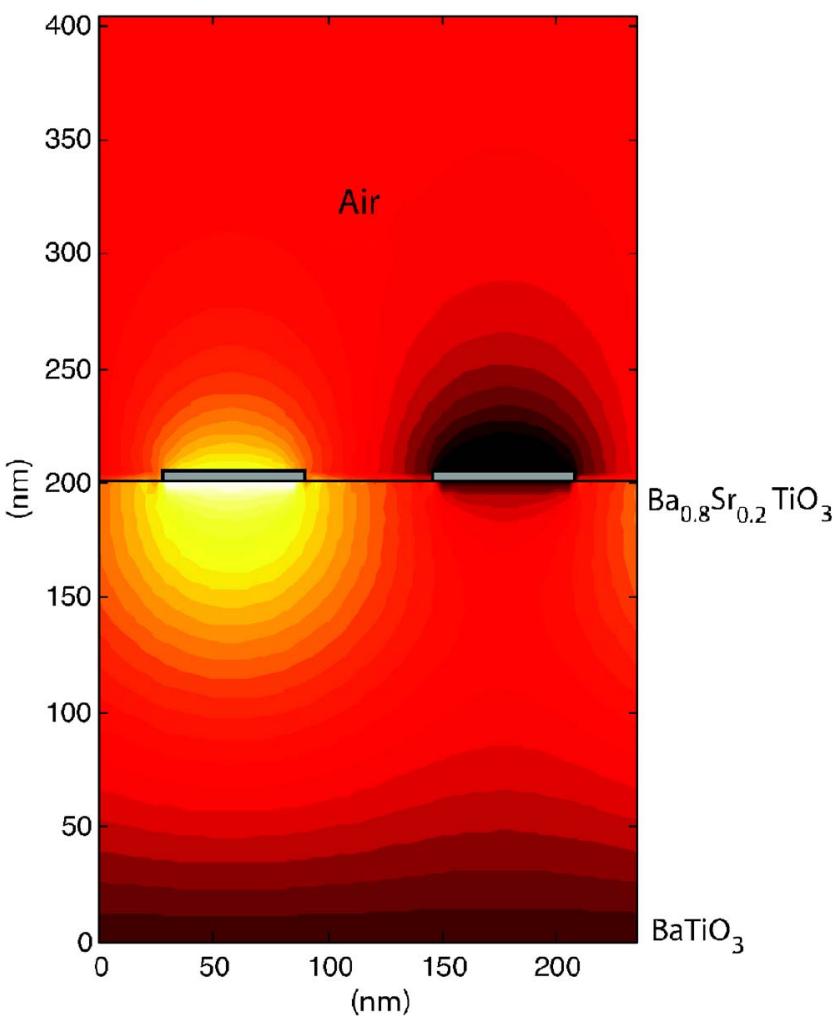

larization decreases, but very slightly, along the $x_{2}$ direction. The compositional gradient does not lead to the polarization gradient expected from the bulk spontaneous polarization of compositions between pure $\mathrm{Ba}_{0.8} \mathrm{Sr}_{0.2} \mathrm{TiO}_{3}$ and $\mathrm{BaTiO}_{3}$ due to the electrostatic interactions. The slight polarization change is accompanied by a large built-in field. This graded structure behaves in effect as a single ferroelectric system instead of an aggregate of different ferroelectric layers. These results directly affect the dielectric properties of the film, making it behave like a homogeneous ferroelectric, as evidenced by Fig. 1 above.

The behavior of the graded BST film in the IDE geometry stands in sharp contrast to the PE case [Fig. 2(b)]. Specifically, the polarization displays a large gradient in the top third of the film, where the grading is present, and there is a negligible built-in field. The result is a broad phase transition with temperature, as shown in Fig. 1, desired for tunable filter applications.

The influence of geometry can also be understood as the difference between series capacitances (PE configuration) and parallel capacitances (IDE configuration). In the IDE parallel capacitance case, a large part of the applied field is across the compositionally graded layers, as evidenced by the potential contours in Fig. 3. In this geometry the field is entirely due to the applied potential on the left surface electrode, unlike the PE case where a large built-in potential develops.

It is worth noting that the parallel plate configuration can be easily converted to a parallel capacitance geometry by changing the grading direction of the film, i.e., changing the composition along the surface of the film, although this may be more difficult to grow experimentally.

In the results detailed above for modestly graded BST in

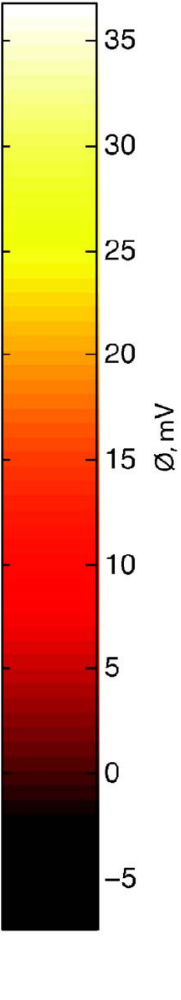

FIG. 3. (Color online) A contour of the calculated room temperature potential due to an applied field on the left electrode of a $200 \mathrm{~nm}$ graded $\mathrm{BaTiO}_{3}-\mathrm{Ba}_{0.8} \mathrm{Sr}_{0.2} \mathrm{TiO}_{3}$ film used in the IDE geometry. the PE geometry, we confirmed that the one-dimensional analysis [Eqs. (6) and (7)] was adequate to capture the dielectric behavior by comparing with two-dimensional calculations performed on the same system. However, when analyzing aggressive polarization gradings, such as $\mathrm{PbTiO}_{3}-$ $\mathrm{BaTiO}_{3}$ (spontaneous polarization $P_{s}$ of 75 and $25 \mu \mathrm{C} / \mathrm{m}^{2}$ respectively at room temperature), we noted switching behavior for the PE geometry that cannot be captured using the simple one-dimensional (1D) model. Figure 4 illustrates the capacitance behavior of a graded $c$-axis $\mathrm{PbTiO}_{3}-\mathrm{BaTiO}_{3}$ thin film between two parallel electrodes. Below $\sim 300{ }^{\circ} \mathrm{C}$, the graded film has a small polarization gradient and a large built-in potential as predicted by the one-dimensional approach (for example, Fig. 2). Above $300{ }^{\circ} \mathrm{C}$, this profile becomes unstable and switches to an $a$-axis film. This transition rids the film of the built-in field and allows a larger polarization gradient, as illustrated in the inset of Fig. 4, which represents the calculated polarization vector field at $326{ }^{\circ} \mathrm{C}$. At higher temperatures the entire film finally transitions into the paraelectric state. These results suggest that switching must be taken into account for films with large polarization grading, as it may strongly influence the overall dielectric response. The simple one-dimensional approach only predicts a ferroelectric-paraelectric phase transition at $300{ }^{\circ} \mathrm{C}$, but not the switching that acts to relieve the film of the large cost of electrostatic interactions between the $c$-axis graded layers.

\section{CONCLUSION}

To conclude, we presented a continuum model that accounts for the spatial variation in properties and the longrange electrostatic interactions in functionally graded ferro- 


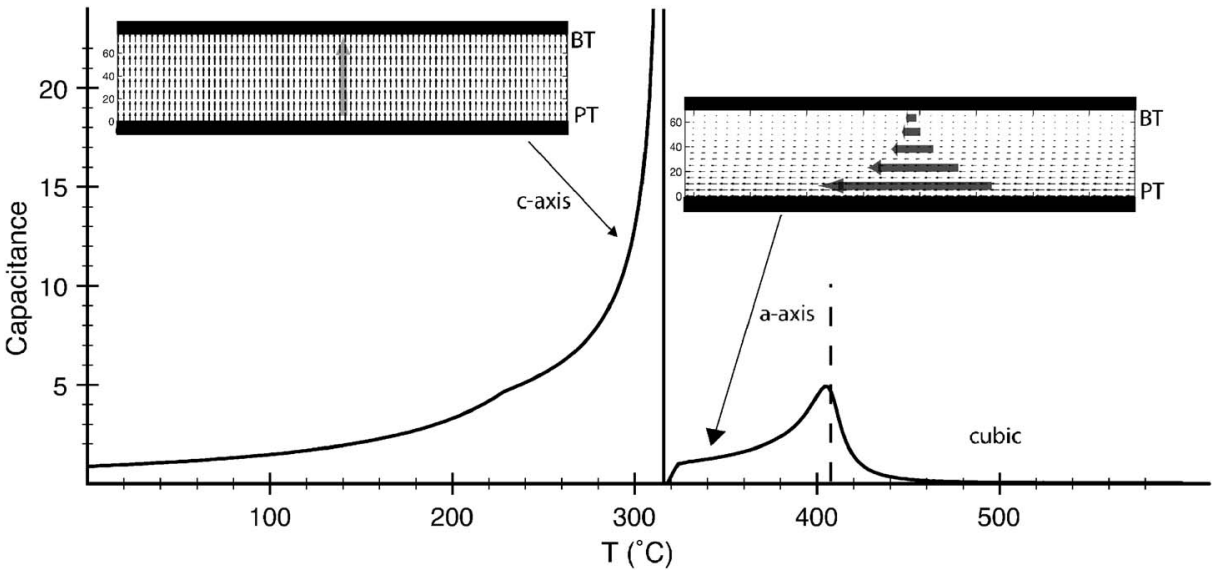

FIG. 4. The dielectric response of a graded $200 \mathrm{~nm} \mathrm{PbTiO}_{3}-\mathrm{BaTiO}_{3}$ thin film. Aggressive compositional grading can lead to switching of polarization components upon applying an electric field, even for the parallel plate electrode geometry, as evidenced by the sample computed polarization vectors before and after $300{ }^{\circ} \mathrm{C}$. Starting with a $c$-axis film and until $\sim 300{ }^{\circ} \mathrm{C}$, a large built-in potential accumulates in the film and a small polarization gradient along the thickness of the film is observed. Switching to an $a$-axis film allows for a larger polarization gradient, until finally the entire film transitions to the cubic state.

electric thin films, with an emphasis on the dielectric behavior. Two geometries are emphasized as case studies: parallel electrode and interdigitated electrode configurations. In both cases, we look for solutions of the polarization nominally aligned with the applied electric field and compute the temperature-dependent dielectric response. We find that the parallel electrode configuration results in a strong temperature dependence of the dielectric constant due to the strong electrostatic interactions between the different layers. On the other hand, interdigitated electrodes lead to a parallel capacitance geometry that results in a broad phase transition with temperature, as desired for tunable filter applications. Figure 5 illustrates the temperature stability and high capacitance of such a device compared to a pure film of $\mathrm{BaTiO}_{3}$, over a wide range of important operating temperatures.

Furthermore, we commented on the applicability of the one-dimensional approach to describe the dielectric behavior of graded ferroelectric films. Aggressive compositional grading can lead to switching, which can be taken into account

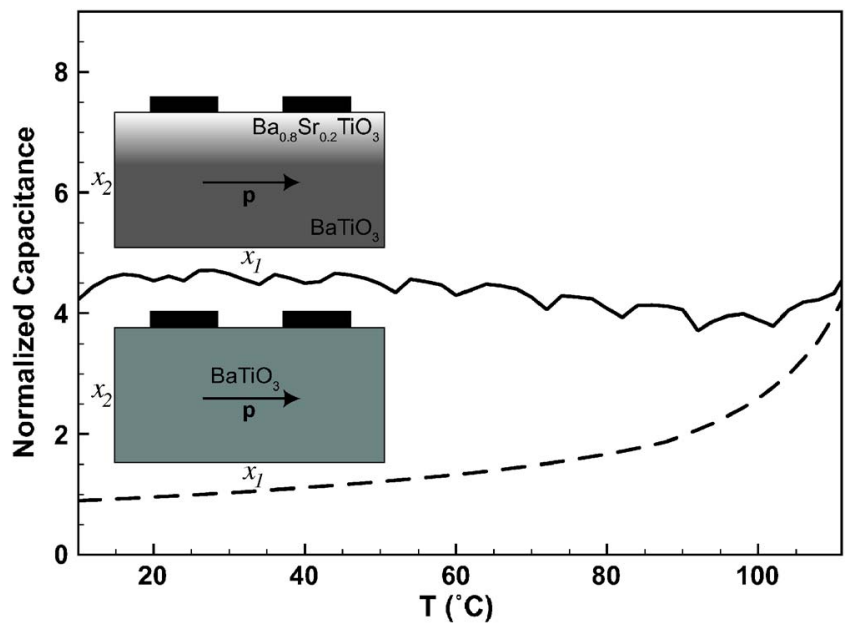

FIG. 5. (Color online) Temperature insensitivity and high capacitance achieved in a graded ferroelectric BST capacitor via interdigitated electrodes. by a two-dimensional model. We expect that these results will be beneficial as design tools for functionally graded tunable devices.

\section{ACKNOWLEDGMENTS}

One of the authors (M.Y.E.) would like to acknowledge an Applied Materials graduate fellowship. This work was partially funded by the U.S. Department of Defense MURI award DAAD19-01-1-0517, administered by the Army Research Office.

${ }^{1}$ D. M. Potrepka, S. Hirsch, M. W. Cole, W. D. Nothwang, S. Zhong, and S. P. Alpay, J. Appl. Phys. 99, 014108 (2006).

${ }^{2}$ M. W. Cole, W. D. Nothwang, C. Hubbard, E. Ngo, and M. Ervin, J. Appl. Phys. 93, 9218 (2003).

${ }^{3}$ B. Acikel, T. R. Taylor, P. J. Hansen, J. S. Speck, and R. A. York, IEEE Microw. Wirel. Compon. Lett. 12, 237 (2002).

${ }^{4}$ J. Serraiocco, B. Acikel, P. Hansen, T. Taylor, H. Xu, J. S. Speck, and R. A. York, Integr. Ferroelectr. 49, 161 (2002).

${ }^{5}$ J. L. Serraiocco, P. J. Hansen, T. R. Taylor, J. S. Speck, and R. A. York, Integr. Ferroelectr. 56, 1087 (2003).

${ }^{6}$ H. T. Xu, N. K. Pervez, and R. A. York, Integr. Ferroelectr. 77, 27 (2005).

${ }^{7}$ X. H. Zhu, N. Chong, H. L. W. Chan, C. L. Choy, K. H. Wong, Z. Liu, and

N. Ming, Appl. Phys. Lett. 80, 3376 (2002).

${ }^{8}$ T. A. Vanderah, Science 298, 1182 (2002).

${ }^{9}$ A. K. Tagantsev, V. O. Sherman, K. F. Astafiev, J. Venkatesh, and N. Setter, J. Electroceram. 11, 5 (2003).

${ }^{10}$ S. G. Lu, X. H. Zhu, C. L. Mak, K. H. Wong, H. L. W. Chan, and C. L. Choy, Appl. Phys. Lett. 82, 2877 (2003).

${ }^{11}$ X. H. Zhu et al., Mater. Sci. Eng., B 118, 219 (2005).

${ }^{12}$ R. Slowak, S. Hoffmann, R. Liedtke, and R. Waser, Integr. Ferroelectr. 24, 169 (1999).

${ }^{13}$ Z. G. Ban, S. P. Alpay, and J. V. Mantese, Phys. Rev. B 67, 184104 (2003).

${ }^{14}$ S. Zhong, S. P. Alpay, Z. G. Ban, and J. V. Mantese, Integr. Ferroelectr. 71, 1 (2005).

${ }^{15}$ Y. C. Shu and K. Bhattacharya, Philos. Mag. B 81, 2021 (2001).

${ }^{16}$ Y. Xiao, Ph.D. thesis, California Institute of Technology, 2004.

${ }^{17}$ W. Zhang and K. Bhattacharya, Acta Mater. 53, 185 (2005).

${ }^{18}$ N. A. Pertsev, A. G. Zembilgotov, and A. K. Tagantsev, Phys. Rev. Lett. 80, 1988 (1998).

${ }^{19}$ G. A. Rossetti, J. P. Cline, and A. Navrotsky, J. Mater. Res. 13, 3197 (1998).

${ }^{20}$ K. Dayal, Ph.D. thesis, California Institute of Technology, 2006.

${ }^{21}$ K. Dayal and K. Bhattacharya, Acta Mater. 2006 (to be published). 